\title{
BMJ Open Mortality following a brain tumour diagnosis in patients with multiple sclerosis
}

\author{
Scott Montgomery, ${ }^{1,2,3}$ Ahmad Hassan, ${ }^{2}$ Shahram Bahmanyar, ${ }^{3,4}$ Ole Brus, ${ }^{1}$ \\ Oula Hussein, ${ }^{1}$ Ayako Hiyoshi, ${ }^{1}$ Jan Hillert, ${ }^{5}$ Tomas Olsson, ${ }^{6}$ Katja Fall ${ }^{1,2}$
}

To cite: Montgomery $S$, Hassan A, Bahmanyar S, et al. Mortality following a brain tumour diagnosis in patients with multiple sclerosis. BMJ Open 2013;3 e003622. doi:10.1136/ bmjopen-2013-003622

- Prepublication history for this paper is available online. To view these files please visit the journal online (http://dx.doi.org/10.1136/ bmjopen-2013-003622).

Received 19 July 2013 Revised 3 October 2013 Accepted 4 October 2013

CrossMark

For numbered affiliations see end of article.

Correspondence to

Professor Scott Montgomery; scott.montgomery@orebroll. se

\section{ABSTRACT}

Objectives: As brain tumours and their treatment may theoretically have a poorer prognosis in inflammatory central nervous system diseases such as multiple sclerosis (MS), all-cause mortality following a brain tumour diagnosis was compared between patients with and without MS. The potential role of age at tumour diagnosis was also examined.

Setting: Hospital inpatients in Sweden with assessment of mortality in hospital or following discharge.

Participants: Swedish national registers identified 20543 patients with an MS diagnosis (1969-2005) and they were matched individually to produce a comparison cohort of 204163 members of the general population without MS. Everyone with a primary brain tumour diagnosis was selected for this study: 111 with MS and 907 without MS.

\section{Primary and secondary outcome measures:}

5-year mortality risk following brain tumour diagnosis and age at brain tumour diagnosis.

Results: A non-statistically significant lower mortality risk among patients with MS (lower for those with tumours of high-grade and uncertain-grade malignancy and no notable difference for low-grade tumours) produced an unadjusted $\mathrm{HR}$ (and $95 \% \mathrm{Cl}$ ) of 0.75 (0.56 to 1.02). After adjustment for age at diagnosis, grade of malignancy, sex, region of residence and socioeconomic index, the HR is $0.91(0.67-1.24)$. The change in estimate was largely due to adjustment for age at brain tumour diagnosis, as patients with MS were on average 4.7 years younger at brain tumour diagnosis than those in the comparison cohort $(p<0.001)$.

Conclusions: Younger age at tumour diagnosis may contribute to mortality reduction in those with highgrade and uncertain-grade brain tumours. Survival following a brain tumour is not worse in patients with MS; even after age at brain tumour diagnosis and grade of malignancy are taken into account.

\section{INTRODUCTION}

It has been reported that brain tumours are diagnosed more frequently in patients with multiple sclerosis (MS) than among the

\section{Strengths and limitations of this study}

- The majority of patients with multiple sclerosis (MS) who had brain tumours during the study period were included and compared with patients with brain tumours who did not have MS.

- Use of all-cause mortality identifies as an unambiguous end-point, but the specific mechanism is not identified.

- Information on brain tumour stage and size at diagnosis was not available.

general population, despite an overall reduction in cancer risk in MS. ${ }^{1}$ An earlier study using the same material as used here identified an increase in the detection of brain tumours of low-grade rather than high-grade malignancy in Swedish patients with MS. Some of the increased frequency of brain tumours may have been due to relatively frequent neuroimaging of the central nervous system (CNS) in patients with MS, resulting in an increased tumour detection rate, including for asymptomatic tumours. ${ }^{1}$

Despite the apparently lower proportion of high-grade brain tumour types in $\mathrm{MS},{ }^{2}$ prognosis following a brain tumour diagnosis in patients with an inflammatory degenerative disease of the CNS remains a concern. It has been suggested that associated treatments including brain surgery, radiotherapy and chemotherapy may be relevant to the course of MS: it is plausible that surgery and radiotherapy result in the liberation of brain-specific antigens that might promote autoimmunity and thus adversely influence the disease course in $\mathrm{MS}^{2}$ We therefore investigated mortality following a first brain tumour diagnosis in patients with MS, compared with people without MS who also had a first brain tumour diagnosis; MS disease course may influence mortality, but this study was not able to investigate this putative mediating factor, and examined mortality as the 
final outcome. Age at first brain tumour diagnosis as an explanatory factor for variation in mortality risk was also investigated.

This study utilises a cohort of Swedish patients with MS and a matched general population comparison cohort. ${ }^{1}$ The analysis presented here is based on the minority subset from each cohort with a primary brain tumour and mortality among these patients is the main outcome.

\section{PATIENTS AND METHODS}

The individuals included in this study were drawn from two larger Swedish cohorts, described previously. ${ }^{1}$ Diagnoses of MS between 1969 and 2005 were identified using the National Inpatient Register ${ }^{3}$ and the National Swedish Multiple Sclerosis Register. ${ }^{4}$ The original study design was that patients with MS were individually matched with 12 individuals (a lower number was achieved in a minority) by year of birth, sex, vital status and region of residence, at the time of diagnosis in the matched case. The Total Population Register provided information on date of death or migration. The National Swedish Cancer Register ${ }^{5}$ identified cancer diagnoses, and brain tumours were classified as high malignancy, low malignancy and uncertain level of malignancy (table 1). A six-category socioeconomic index was based on occupation identified from the census nearest in time to study entry (manual workers, non-manual workers, professionals, self-employed, farmers and others).

From among the original 20543 with and 204163 without MS, we excluded 479 (267 with MS) due to missing information. We then identified all those with a brain tumour who did not have a prior cancer diagnosis at any other site. A total of 111 with MS and 907 without had a primary brain tumour diagnosis and comprises the main study population examined here. A further post hoc analysis extended the sample to those with either a primary brain tumour or a primary spinal cord tumour, increasing numbers to 124 with MS and 949 without MS.

\section{Statistical analysis}

Cox regression was used to estimate mortality risk in the 5 years following brain tumour diagnoses in patients with MS compared with the reference cohort. Follow-up was from brain tumour diagnosis to death, emigration, 5 years duration or 31 December 2005, whichever occurred first. Adjustment was for age at brain tumour diagnosis (continuous), level of malignancy, sex, geographical region (Northern, Central and Southern Sweden) and socioeconomic index. Further analyses were stratified by grade of malignancy. Mortality risk for 10 years was also examined and a Kaplan-Meier curve was produced. The proportionality of hazards assumption was verified for all analyses.

Table 1 Brain tumour distribution

\begin{tabular}{|c|c|c|c|}
\hline \multirow[b]{2}{*}{ Tumour classificationl } & \multirow[b]{2}{*}{ PAD code } & \multicolumn{2}{|c|}{ Number of participants $(\mathrm{N}=1008)$} \\
\hline & & $\begin{array}{l}\mathrm{MS} \\
\mathrm{N}=110(\%)\end{array}$ & $\begin{array}{l}\text { No MS } \\
\mathrm{N}=898 \text { (\%) }\end{array}$ \\
\hline \multicolumn{4}{|l|}{ Low grade malignancy } \\
\hline Plexiform neurofibroma/hamartoma & 451 & $7(6.4)$ & $52(5.8)$ \\
\hline Meningioma, benign & 461 & $33(30.0)$ & $251(28.0)$ \\
\hline Astrocytoma (grades 0-2) & 475 & $10(9.1)$ & $130(14.5)$ \\
\hline Ependymoma (grades 1-2) & 485 & $2(1.8)$ & $9(1.0)$ \\
\hline Hemangioblastoma & 511 & $1(0.9)$ & $6(0.7)$ \\
\hline Dermoid cyst, cholesteatoma, teratoma & 821 & $0(0)$ & $2(0.2)$ \\
\hline Craniopharyngioma & 881 & $3(2.7)$ & $8(0.9)$ \\
\hline Germinoma & 981 & $2(1.8)$ & $2(0.2)$ \\
\hline \multicolumn{4}{|l|}{ High-grade malignancy } \\
\hline Neuroblastoma & 416 & $0(0)$ & $4(0.5)$ \\
\hline Medulloblastoma & 436 & $1(0.9)$ & $4(0.5)$ \\
\hline Neurinoma & 456 & $1(0.9)$ & $1(0.1)$ \\
\hline Astrocytoma (grades 3-4) & 476 & $28(25.5)$ & $314(35.0)$ \\
\hline Ependymoblastoma (grades 3-4) & 486 & $1(0.9)$ & $0(0)$ \\
\hline Meningioma & 466 & $1(0.9)$ & $9(1.0)$ \\
\hline Teratoma & 826 & $0(0)$ & $1(0.1)$ \\
\hline \multicolumn{4}{|l|}{ Uncertain grade of malignancy } \\
\hline Neurinoma & 453 & $0(0)$ & $1(0.1)$ \\
\hline Hemangioma & 501 & $2(1.8)$ & $13(1.5)$ \\
\hline Neuroepithelial tumour & 991 & $10(9.1)$ & $42(4.7)$ \\
\hline Hemangiopericytoma, NOS & 533 & $0(0)$ & $1(0.1)$ \\
\hline Other & & $8(7.3)$ & $48(5.3)$ \\
\hline
\end{tabular}


To examine whether the results are influenced substantially by immunomodulatory therapy, the follow-up period was right truncated to end before 1996, when the first of the more recent therapies, interferon $\beta$, was introduced. ${ }^{6}$

A further post-hoc analysis included patients with a primary spinal cord tumour, in addition to those with primary brain tumours.

To investigate variation in age at brain tumour diagnosis, age was the dependent variable in linear regression analysis. Adjustment was for level of malignancy, sex, geographical region and socioeconomic index.

The analysis used SPSS statistical software, version 21.

\section{RESULTS}

Table 1 reports the distribution of brain tumour types in those with and without MS. As previously reported, ${ }^{2}$ a lower proportion of those with MS had tumours of highgrade malignancy compared with the non-MS cohort. The proportions in the three broad grade categories (high, low and uncertain malignancy) are also shown in table 2. Table 2 reports other characteristics of the study population. There was a lower mortality risk in the 5 years following brain tumour diagnosis among patients with MS, which approached statistical significance before, but not after adjustment (table 2 and figure 1). Adjustment for age at cancer diagnosis accounts for the change in estimates. Figure 1 demonstrates that a similar pattern of association continues after 5 years of follow-up.

When stratified by grade of malignancy, there is no difference in mortality by MS for the low-grade malignancy group. There is a lower mortality risk in patients with MS among those with high-grade and undetermined-grade tumours. The association was observed to be statistically significant among patients with undetermined-grade tumours, but adjustment for the potential confounding factors eliminated this significance. Although not statistically significant, a lower mortality risk remained among patients with MS.

Right truncation of the follow-up period to before 1996 did not alter the findings notably, producing unadjusted and adjusted HRs (with 95\% CIs) for the association of MS with mortality of 0.67 ( 0.46 to 0.98 ; $\mathrm{p}=0.037$ ) and 0.79 (0.54 to $1.16 ; \mathrm{p}=0.234)$, respectively.

Addition of patients with a primary spinal cord tumour to the main analysis did not alter the results dramatically, producing unadjusted and adjusted HRs for mortality associated with MS of $0.68 \quad(0.50-0.93$; $\mathrm{p}=0.014)$ and $0.80(0.58-1.09 ; \mathrm{p}=0.161)$, respectively.

Linear regression indicated patients with MS were on average 4.7 years younger at brain tumour diagnosis, after adjustment for the potential confounding factors $(\mathrm{p}<0.001)$.

Table 2 Five-year mortality following a brain tumour diagnosis among patients with and without multiple sclerosis (MS)

\begin{tabular}{|c|c|c|c|c|c|c|}
\hline & \multicolumn{2}{|c|}{ Events/patients (\%) } & \multicolumn{2}{|l|}{ Unadjusted } & \multicolumn{2}{|l|}{ Adjusted* } \\
\hline & $\begin{array}{l}\mathrm{MS} \\
\mathrm{N}=110(10.9 \%)\end{array}$ & $\begin{array}{l}\text { No MS } \\
\mathrm{N}=898(89.1 \%)\end{array}$ & HR $(95 \% \mathrm{Cl})$ & p Value & $\mathrm{HR}(95 \% \mathrm{Cl})$ & p Value \\
\hline \multicolumn{7}{|l|}{ Unstratified } \\
\hline \multicolumn{7}{|l|}{ Cohort } \\
\hline No MS & - & $478 / 898$ & Reference & & & \\
\hline MS & $45 / 110$ & - & 0.75 (0.56 to 1.02$)$ & 0.070 & $0.91(0.67$ to 1.24$)$ & 0.554 \\
\hline Age (years) & $49.6 \dagger$ & $52.3 †$ & $1.04(1.04$ to 1.05$)$ & $<0.001$ & 1.05 (1.04 to 1.05$)$ & $<0.001$ \\
\hline \multicolumn{7}{|l|}{ Sex } \\
\hline Male & $25 / 44(40.0)$ & 177/283 (31.5) & Reference & & Reference & \\
\hline Female & 20/66 (60.0) & $301 / 615(68.5)$ & $0.65(0.55$ to 0.78$)$ & $<0.001$ & $0.89(0.74$ to 1.07$)$ & 0.201 \\
\hline \multicolumn{7}{|c|}{ Brain tumour histological classification } \\
\hline Low grade & $17 / 58(52.7)$ & $131 / 460(51.2)$ & Reference & & Reference & \\
\hline High grade & 22/32 (29.1) & 284/333 (37.1) & 4.76 (3.89 to 5.82$)$ & $<0.001$ & 5.11 (4.14 to 6.31$)$ & $<0.001$ \\
\hline Uncertain grade & $6 / 20(18.2)$ & $63 / 105(11.7)$ & 2.44 (1.83 to 3.25$)$ & $<0.001$ & $2.47(1.85$ to 3.30$)$ & $<0.001$ \\
\hline \multicolumn{7}{|c|}{ Stratified by brain tumour histology } \\
\hline \multicolumn{7}{|l|}{ Low grade } \\
\hline No MS & - & $131 / 460$ & Reference & & Reference & \\
\hline MS & $17 / 58$ & - & $1.10(0.66$ to 1.82$)$ & 0.722 & $1.14(0.68$ to 1.89$)$ & 0.618 \\
\hline \multicolumn{7}{|l|}{ High grade } \\
\hline No MS & - & $284 / 333$ & Reference & & Reference & \\
\hline MS & $22 / 32$ & - & $0.75(0.49$ to 1.16$)$ & 0.200 & 0.91 (0.58 to 1.42$)$ & 0.665 \\
\hline \multicolumn{7}{|l|}{ Uncertain grade } \\
\hline No MS & - & $63 / 105$ & Reference & & Reference & \\
\hline MS & $6 / 20$ & - & $0.39(0.17$ to 0.89$)$ & 0.026 & $0.67(0.27$ to 1.68$)$ & 0.394 \\
\hline
\end{tabular}




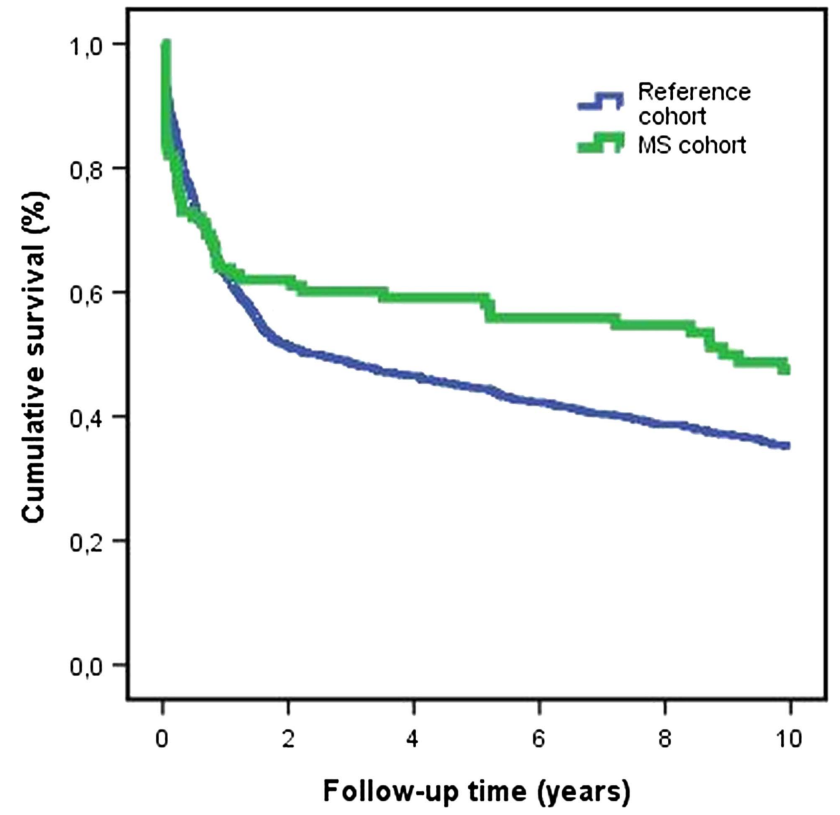

Figure 1 Mortality following a brain tumour diagnosis among patients with and without multiple sclerosis. Patients with brain tumours were followed for up to 10 years from their diagnosis and treatment. The Kaplan-Meier curves indicate mortality risk, divided into those with and without multiple sclerosis.

\section{DISCUSSION}

This study compared Swedish patients with MS who received a primary brain tumour diagnosis between 1969 and 2005, with MS-free members of the general population also with a primary brain tumour. Patients with MS who have brain tumours did not have a raised mortality risk compared with brain tumour patients without MS. The patients with MS tended to have fewer high-grade malignancies as previously described, ${ }^{2}$ and on average were diagnosed at an earlier age. Even after taking into account age at tumour diagnosis and grade of malignancy, there is no evidence of a further raise in mortality risk associated with brain tumours in patients with MS.

It is plausible that brain tumours may have a worse prognosis in patients with an inflammatory disease of the CNS and it has been suggested that treatment for brain tumours may have adverse implications for MS disease course. ${ }^{2}$ Although we were unable to examine alterations in MS disease course here and did not have information on changes in MS phenotype, we assessed mortality as a possible consequence. Mortality is a plausible outcome as patients with MS have a higher risk of fatal comorbidity from a variety of causes including infections $^{7}$ and cardiovascular disease. ${ }^{8}$ In general, patients with MS may have shorter life expectancy than the general population ${ }^{9}{ }^{10}$ with an average of approximately 5-10 years of life lost. ${ }^{11}$ Mortality has been identified as an unambiguously valid outcome relevant to MS severity and disease progression, including through causes of death such as suicide which is more common among patients with MS, thus emphasising the importance of considering all-cause mortality as the outcome. ${ }^{12}$ The results of our analyses indicate that brain tumours and associated treatment are not greater mortality risks in patients with MS than in patients without MS who have brain tumours. When the analysis was extended to include patients with primary spinal cord tumours, as well as brain tumours, the results were not notably altered and did not suggest an increased mortality risk among patients with MS.

Earlier age at brain tumour diagnosis appears to help explain some of the observed lower mortality risk, consistent with more frequent neuroimaging in patients with MS enhancing tumour detection rates. While this will result in a higher diagnosis rate for the asymptomatic tumours of low-grade malignancy reported here and as previously described, ${ }^{2}$ it will also result in an earlier diagnosis of high-grade malignant tumours: this is consistent with evidence of reduction in mortality risk for patients with MS with high-grade and uncertain-grade tumour types. The magnitude of the inverse association is attenuated by the adjustment for age at diagnosis. Another non-mutually exclusive possibility is that brain tumour onset occurs at a younger age among patients with MS. Despite the role of detection or onset at a younger age, there was still no evidence of increased mortality risk in patients with MS, even after earlier age at diagnosis and tumour grade were taken into account through statistical adjustment.

Although it is not possible to provide a precise proportion, we believe this study will have identified the majority of patients with MS with a primary brain tumour diagnosed and treated during the study period. The patient register identified inpatient diagnoses for most of the study period (with outpatient diagnoses from 2001), and some patients with MS who were not inpatients will not have been included in the larger original MS cohort. However, as the cancer register is over $99 \%$ complete, virtually all brain tumour diagnoses will have been recorded and most of these patients will have been admitted to hospital for treatment. This will have resulted in comorbid diagnoses, such as MS, being included in the patient register and thus the likely inclusion of such patients in our MS cohort.

Potential limitations of this study include lack of knowledge about tumour stage or size at diagnosis, but the findings are consistent with earlier stage and smaller tumour size in patients with MS due to earlier detection. It was not possible to assign a malignancy grade to all tumours, but some reduction of mortality risk was seen in the high-grade as well as uncertain grade categories. We restricted the main results to deaths that occurred during the first 5 years after diagnosis when tumourrelated adverse sequelae are most likely to occur; but the findings were similar for longer follow-up. To help explain the results it would have been useful to examine MS disease course in greater detail, particularly changes in MS phenotype: unfortunately such longitudinal 
information on phenotype was not available to us. It was not possible to examine influences of more recently introduced immunomodulatory therapy, as these data were not available from the registers for this study period. To tackle this, we performed a post hoc analysis with right truncation, so that follow-up was ended prior to introduction of the first of such therapies, interferon $\beta$, which began to be used in Sweden in $1996 .{ }^{6}$ While this does not directly test the possible influence of these therapies, the results show that they did not have a major influence on the overall findings. Another potential limitation is our lack of access to reliable information on the number of MRI scans performed. While we hypothesise that such procedures may enhance earlier tumour detection in patients with MS, we cannot confirm this using these data.

Earlier detection due to more frequent MS-related CNS investigations may contribute to reduced mortality risk for high-grade tumours. Even after age at diagnosis and malignancy grade are taken into account, brain tumours in patients with MS are not associated with higher mortality than in patients without MS, indicating that neither tumours nor their treatment are more likely to be fatal in this patient group.

\section{Author affiliations \\ ${ }^{1}$ Clinical Epidemiology and Biostatistics, Örebro University Hospital, Örebro, Sweden \\ ${ }^{2}$ School of Health and Medical Sciences, Örebro University, Örebro, Sweden \\ ${ }^{3}$ The Clinical Epidemiology Unit and Pharmacoepidemiology Unit, Karolinska Institutet, Stockholm, Sweden \\ ${ }^{4}$ Faculty of Medicine, Golestan University of Medical Sciences, Gorgan, Iran \\ ${ }^{5}$ Department of Clinical Neuroscience, Karolinska University Hospital Huddinge, Karolinska Institutet, Stockholm, Sweden \\ ${ }^{6}$ Neuroimmunology Unit, Department of Medicine, Karolinska University \\ Hospital, Karolinska Institutet, Stockholm, Sweden}

Contributors SM, AHa and KF developed the study concepts and hypotheses. $\mathrm{OB}, \mathrm{OH}$ and $\mathrm{AHi}$ conducted the statistical analysis with input from $\mathrm{AHi}$. SB extracted and prepared data from the original cohorts. SB, TO, JH and SM created the original cohorts, which provided these data. SM and AHa created the first draft of the manuscript, which was critically edited by all the authors. $\mathrm{OH}$ created the tables, with input from $\mathrm{AHa}, \mathrm{AHi}$ and $\mathrm{OB}$.

Funding This work was supported by an Örebro University strategic funding grant.
Competing interests None.

Ethics approval Karolinska Institutet regional ethics committee.

Provenance and peer review Not commissioned; externally peer reviewed.

Data sharing statement We do not have ethical permission to share our data with other researchers. However, the register data we used can be applied for by other researchers if the appropriate ethical permission has been obtained.

Open Access This is an Open Access article distributed in accordance with the Creative Commons Attribution Non Commercial (CC BY-NC 3.0) license, which permits others to distribute, remix, adapt, build upon this work noncommercially, and license their derivative works on different terms, provided the original work is properly cited and the use is non-commercial. See: http:// creativecommons.org/licenses/by-nc/3.0/

\section{REFERENCES}

1. Bahmanyar S, Montgomery SM, Hillert J, et al. Cancer risk among patients with multiple sclerosis and their parents. Neurology 2009;72:1170-7.

2. Hofer S, Linnebank M, Weller M, et al. Correspondence: cancer risk among patients with multiple sclerosis and their parents. Neurology 2010;74:614-15.

3. National Board of Health and Welfare. The Swedish Hospital Discharge Register 1987-1996: Quality and Contents. Stockholm: Centre for Epidemiology, 1998. http://www.socialstyrelsen.se/Lists/ Artikelkatalog/Attachments/8708/2008-125-1_20081251_rev.pdf (accessed 27 Sep 2013).

4. Andersen O. From the Gothenburg cohort to the Swedish multiple sclerosis registry. Acta Neurol Scand Suppl 2012;126:13-19.

5. Mattsson B, Wallgren A. Completeness of the Swedish Cancer Register. Non-notified cancer cases recorded on death certificates in 1978. Acta Radiol Oncol 1984;23:305-13.

6. Andersson A, Persson PM, Fredrikson S. Place of residence determines access to interferon-beta therapy in MS. Less than 15 per cent of patients are treated-big differences between the counties. Lakartidningen 1999;96:5492-5.

7. Montgomery S, Hillert J, Bahmanyar S. Hospital admission due to infections in multiple sclerosis patients. Eur J Neurol 2013;20: 1153-60.

8. Jadidi E, Mohammadi M, Moradi T. High risk of cardiovascular diseases after diagnosis of multiple sclerosis. Multiple Scleroris 2013;19:1336-40.

9. Hirst C, Swingler R, Compston DAS, et al. Survival and cause of death in multiple sclerosis: a prospective population-based study. J Neurol Neurosurg Psychiatry 2008;79:1016-21.

10. Koch-Henriksen N, Bronnum-Hansen H, Stenager E. Underlying cause of death in Danish patients with multiple sclerosis: results from the Danish Multiple Sclerosis Registry. J Neurol Neurosurg Psychiatry 1998;65:56-9.

11. Ragonese $P$, Aridon $P$, Salemi $G$, et al. Mortality in multiple sclerosis: a review. Eur J Neurol 2008;15:123-7.

12. Scalfari A, Knappertz V Cutter $G$, et al. Mortality in patients with multiple sclerosis. Neurology 2013;81:184-92. 